

\title{
Integrated Flight and Propulsion Distributed Control System Simulation Platform Design
}

\author{
Jingbo Peng ${ }^{1}$ \\ Air Force Engineering University \\ $X i$ 'an, 710038, China \\ E-mail: pjb12090126.com \\ Yu Zhang ${ }^{2}$ \\ Air Force Engineering University \\ $X i$ 'an, 710038, China \\ E-mail:zhangyu972840126.com
}

In order to solve the simulation problem of integrated flight propulsion control system based on the distributed structure. A simulation system based on MATLAB/Simulink is designed in this paper. The IFPC system structure is analyzed firstly. The overall structure and all separate modules of the simulation platform which based on CAN bus is then designed by using Truetime simulation toolbox. In order to verify the effectiveness of the designed platform, the performance of distributed integrated flight and propulsion control system with network induced delay and packet dropout is simulated. The result shows that the designed platform can effectively reflect the influence of network characteristics on the system performance. The designed simulation platform is advanced in its multiple adjustable parameters, open access and easy operation, which is an ideal distributed control system simulation platform.

CENet2017

22-23 July, 2017

Shanghai, China

\footnotetext{
${ }^{1}$ Speaker,This study is supported by National Natural Science Foundation of China (51506221)

${ }^{2}$ Correspongding Author
} 


\section{Introduction}

The traditional flight control system and the propulsion control system run separately, but the coupling of the two systems does exist, which makes the control system to sacrifice some stability [1]. As a result, with the development of both platform, it is of great necessity to control both system in an integrate manner [1-3].

The integrated flight and propulsion control (IFPC) is characterized by its great number of supervision and control signals as well as the interconnection of the signals. The traditional point-to-point data transmission is no longer suitable to this kind of system. Distribute control system (DCS) has become a promising new control structure based on the quick development of network technology. The data bus is used in DCS to establish connection among sensors, controllers and actuators. In DCS, some unimportant functions are not completed by the central controller but the smart components, which results in better performance and higher reliability of the whole system. Recently, the development of aero-engine distributed control system is remarkable and fruitful [4-6]. It is expected that distributed control will also become a great choice of the future IFPC systems.

One of the most important aspects of the research on distributed integrated flight and propulsion control (DIFPC) system is the control effectiveness when network factors, like network induced delay and packet dropout, exist. Obviously, it is very costly and risky to construct the bus network and carry out experiments on real aircrafts; therefore, it is of great significance to carry out research on controlling problems when the network factors exist through simulation. The work of this paper focuses on the simulation system design of DIFPC.

\section{Truetime Based DIFPC Simulation Platform Design}

\subsection{Truetime Networked Control System Simulation Tool}

Truetime is a Matlab/Simulink based programming toolbox compatible with Visual C+ +6.0 and its higher editions. Both Matlab and Visual $\mathrm{C}++$ can be used by this toolbox to design different types of networked models, which we believe is an ideal simulation platform for the DIFPC system. The toolbox has four modules, specifically, the network communication module, the real-time inner core module, the battery module and the wireless network module. The parameters of all modules can be set in the dialog box. The code functions are integrated in the real-time inner core module, which can realize programming through Matlab or Visual $\mathrm{C}++$ to simulate the specific functions that the module need to have. The data sending and receiving of different network nodes will obey the pre-determined FP, RM, DM or EDF scheduling strategy, or obey the user-given priority. As the parameters like data transmission rate or the packet dropout rate can directly be set in the dialog box, and the network induced delay can be set in the code functions, and the priority can be designed, which gives the designer higher authority and convenience to carry out research on system performance, scheduling strategy and control simulation.

\subsection{Simulation System Design}

According to the hierarchical, decentralized program, DIFPC system model can be established based on a certain type of aircraft. The inlet control uses open-loop control according to Ma. In the nozzle control, two deflection angle controllers are reserved for the 
future vector nozzle but will not be used at present, which means the deflection angle in this work will be considered unchangeable. The overall structure can be seen in Fig. 1 .

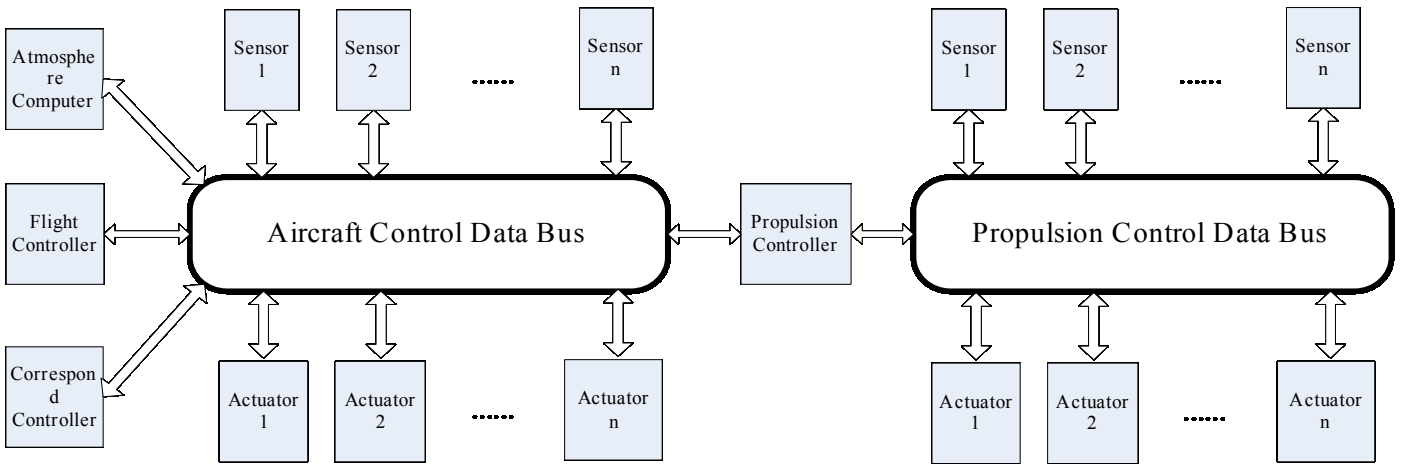

Figure 1: Structure of DIFPC System

In order to use the same communication protocol, the subsystems established in this paper all use CAN bus. The number of smart sensors and smart actuators used in the systems is cut, which means only the control signal related smart components are kept in the system. To further simplify the system, multi-redundant sensors are taken as single-redundant sensors. The simplified CAN bus based DIFPC system Simulink simulation platform is shown in Fig. 2 and Fig. 3.

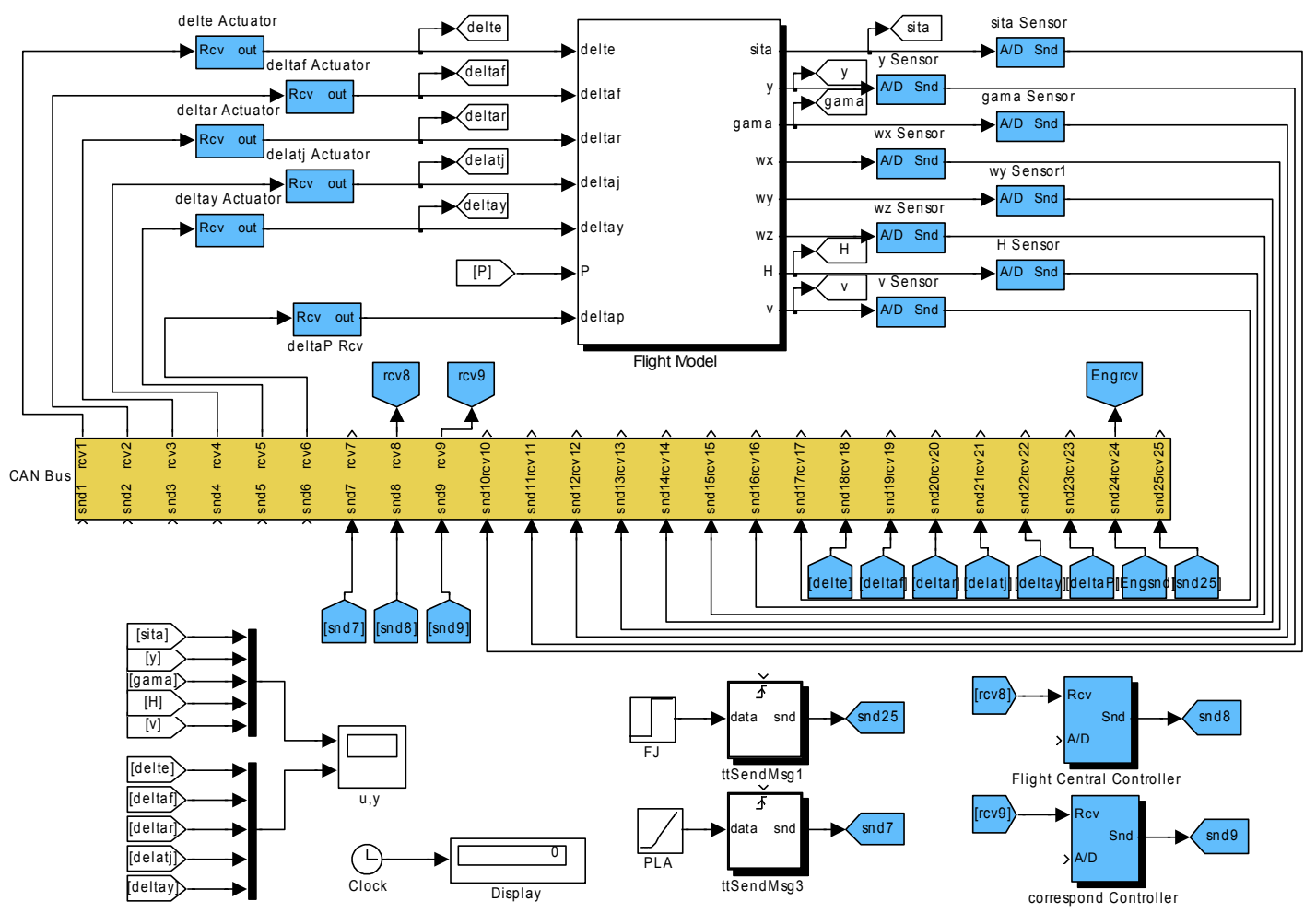

Figure 2 DIFPC Truetime Simulation Platform (Subsystem of Flight Control) 


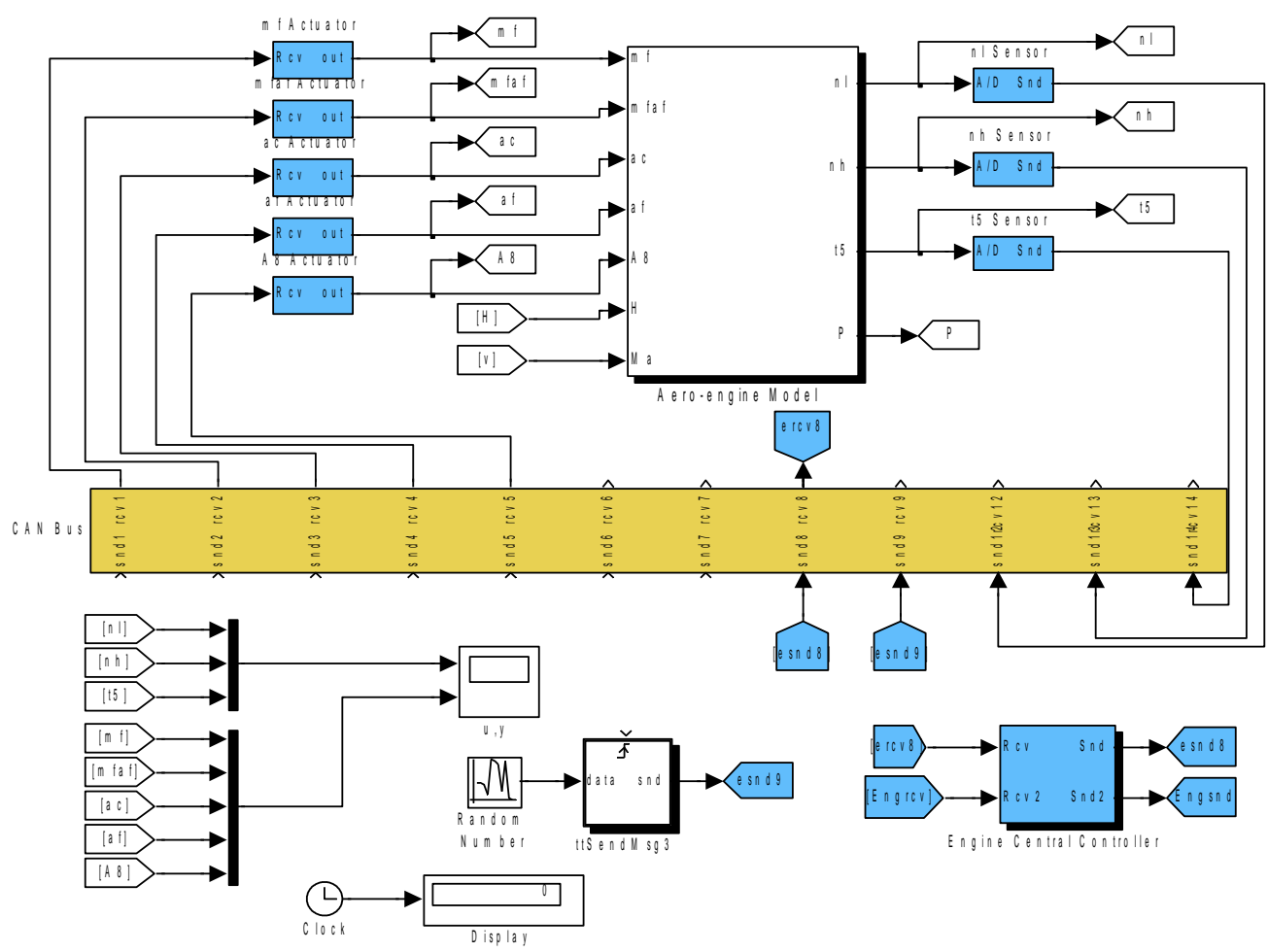

Figure 3 DIFPC Truetime Simulation Platform (Subsystem of Engine Control)

The simplified simulation system consists of 2 network modules, 1 flight central controller, 1 engine central controller, 1 correspond controller, 11 smart sensors (altitude $H$, velocity $v$, pitching angle $\theta$, roll angle $y$, track angle $\gamma, w_{x}$ angular rate, $w_{y}$ angular rate, $w_{z}$ angular rate, high pressure rotor speed $n_{H}$, low pressure rotor speed $n_{L}$, turbo exit temperature $T_{5}$ ), 11 smart actuators (horizontal tail deflection angle $\delta_{e}$, aileron deflection angle $\delta_{f}$, rudder deflection angle $\delta_{r}$, flap $\delta_{j}$, main combustor fuel flow $m_{f}$, afterburning combustor fuel flow $m_{f a f}$, low pressure compressor guide vane deflection angle $\alpha_{F}$, high pressure compressor stator vane deflection angle $\alpha_{C}$, nozzle critical area $\theta_{A \delta}$ ), 2 data sending nodes (control pillar force or displacement $F_{j}$, throttle lever displacement $P L A$ ), 1 bus communication disturbance node (ttSendMsg), 1 flight model module, 1 aeroengine model module and 2 display modules.

Low pressure compressor guide vane deflection angle $\alpha_{F}$ and high pressure compressor stator vane deflection angle $\alpha_{C}$ are open-loop controlled according to the rotor speed and it is believed that it will adjust itself according to certain dynamic characteristics.

The definition of all modules are given in Table 1(limited by the length of the article, the detailed designing process is not given herein).

\begin{tabular}{|l|l|l|}
\hline Module/node & Matlab/Truetime module & Function \\
\hline Network module & Truetime Network module & $\begin{array}{l}\text { Information transmission, node number and packet } \\
\text { dropout rate set, random time delay simulation in } \\
\text { code function, analyzing the influence of network } \\
\text { factors on system performance }\end{array}$ \\
\hline $\begin{array}{l}\text { Smart sensor } \\
\text { module }\end{array}$ & Truetime Kernel module & $\begin{array}{l}\text { Connected with the output access of flight dynamic } \\
\text { model and aero-engine model, collecting data and }\end{array}$ \\
\hline
\end{tabular}




\begin{tabular}{|l|l|l|}
\hline Module/node & Matlab/Truetime module & Function \\
\hline $\begin{array}{l}\text { Smart actuator } \\
\text { module }\end{array}$ & Truetime Kernel module & $\begin{array}{l}\text { realizing fault detection simulation of sensors } \\
\text { model and aero-engine model, driving actuator } \\
\text { according to the CAN bus control signals, carrying } \\
\text { out locally closed-loop control }\end{array}$ \\
\hline $\begin{array}{l}\text { Central controller } \\
\text { module }\end{array}$ & Truetime Kernel module & Realizing control strategy \\
\hline $\begin{array}{l}\text { Flight dynamic } \\
\text { model }\end{array}$ & $\begin{array}{l}\text { Self-packaged Simulink } \\
\text { module }\end{array}$ & $\begin{array}{l}\text { Flight vertical model packaged, input \& output } \\
\text { access }\end{array}$ \\
\hline $\begin{array}{l}\text { Aero-engine model } \\
\text { module }\end{array}$ & $\begin{array}{l}\text { Self-packaged Simulink } \\
\text { module }\end{array}$ & $\begin{array}{l}\text { Aero-engine model packaged, input \& output } \\
\text { access }\end{array}$ \\
\hline Data sending node & Truetime ttSendMsg module & Simulating the pilot operation \\
\hline $\begin{array}{l}\text { Bus communication } \\
\text { disturbance node }\end{array}$ & Truetime ttSendMsg module & $\begin{array}{l}\text { Simulating the disturbances existing in the bus } \\
\text { communication and will send random disturbance } \\
\text { signals to itself during operation }\end{array}$ \\
\hline Display module & $\begin{array}{l}\text { Simulink Scope module and } \\
\text { Mux module }\end{array}$ & Display the controlling variables and state variables \\
\hline
\end{tabular}

Table 1:Definition and Function of Modules in the Simulation Platform

\section{Time Delay Analysis Simulation Example}

In order to testify effectiveness of the designed DIFPC simulation platform, especially the influence of networked factors on control effectiveness, simulations are carried out for the DIFPC system with uncertain time delay and packet dropout. It's previously set that all nodes use invariable priority PrioFP scheduling strategy and the sensors are time-driven with the sampling time of $0.02 \mathrm{~s}$. The state feedback control law $\Delta u(k)=K x(k)$ is adopted by the controller and the IFPC model (the linear quadratic controller designing method mentioned in Reference [7-8] is used to design the state feedback controller). The control variable of the IFPC system is $u=\left[\delta_{c}, m_{f c}, A_{g}\right]$, and the state variable ia $x=\left[v, \alpha, q, \theta, H, n_{L}, n_{H}, m_{f}\right], m_{f c}$ is the controller output . Matlab LQR designer is adopted to obtain the linear quadratic optimal controller, which is given as follows:

$$
\boldsymbol{K}=\left[\begin{array}{cccccccc}
-0.0008 & -0.0009 & -0.0007 & -0.0028 & -0.0394 & 0.0021 & 0.0000 & 1.7384 \\
-0.0000 & 0.0000 & 0.0000 & 0.0000 & -0.0005 & 0.0000 & 0.0000 & 0.0189 \\
0.0000 & -0.0000 & -0.0000 & -0.0000 & 0.0001 & -0.0000 & -0.0000 & -0.0017
\end{array}\right]
$$

To generate uncertain time delay in the control loop, uncertain time delay of various ranges are added to the task function of different nodes. It needs to be pointed out that this time delay generating way is different from that of the actual distributed control system; in the actual system, the network induced delay mainly comes from the waiting time, but here the sending time is taken as the source of time delay, which will not exert influence on the simulation results.

To obtain the system performance when the network induced delay exists, the packet dropout rate of both network modules are set as 0 . The time delay of the nodes is randomly set within the range $\tau \in[T, 2 T]$. The step signal input is added to the loop and system response curve is shown in Fig. 4 (for the length limitation, only several parameters are given here). The 
parameters in the figures are all dimensionless quantities. As indicatd by the simulation results, the system shows a slight trend of instability because of existence of the time delay and some parameter response curves have slight chattering. However, as the time delay was still small, the overall performance of the system is stable and the influence of time delay on the system performance is limited. Then, set the packet dropout rate of network modules as 0.2 and the time delay of the nodes are randomly set within the range $\tau \in[T, 2 T]$. Also add the step signal input to system, and the response curve is shown in Fig. 5. As indicated by the simulation results, when the packet dropout was added to the system, the system performance deteriorated quickly and became more unstable.
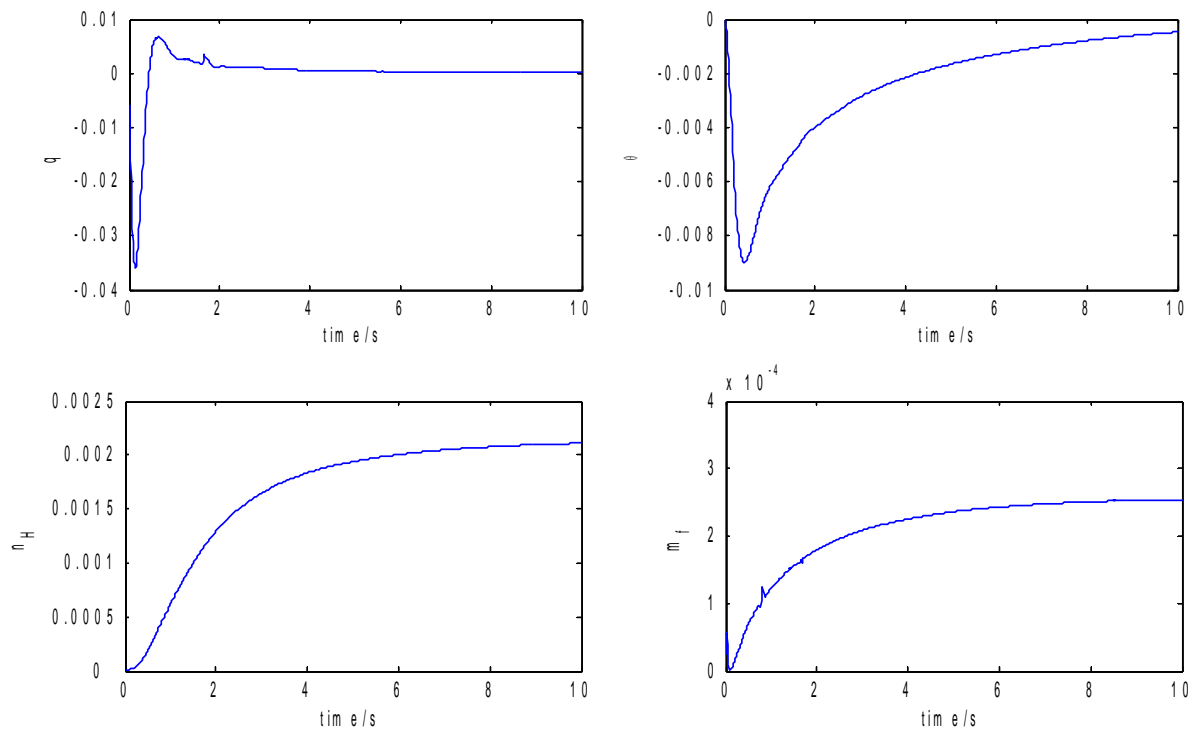

Figure 4 System step input response when the random time delay is $\tau \in[T, 2 T]$ and the packet dropout rate is 0
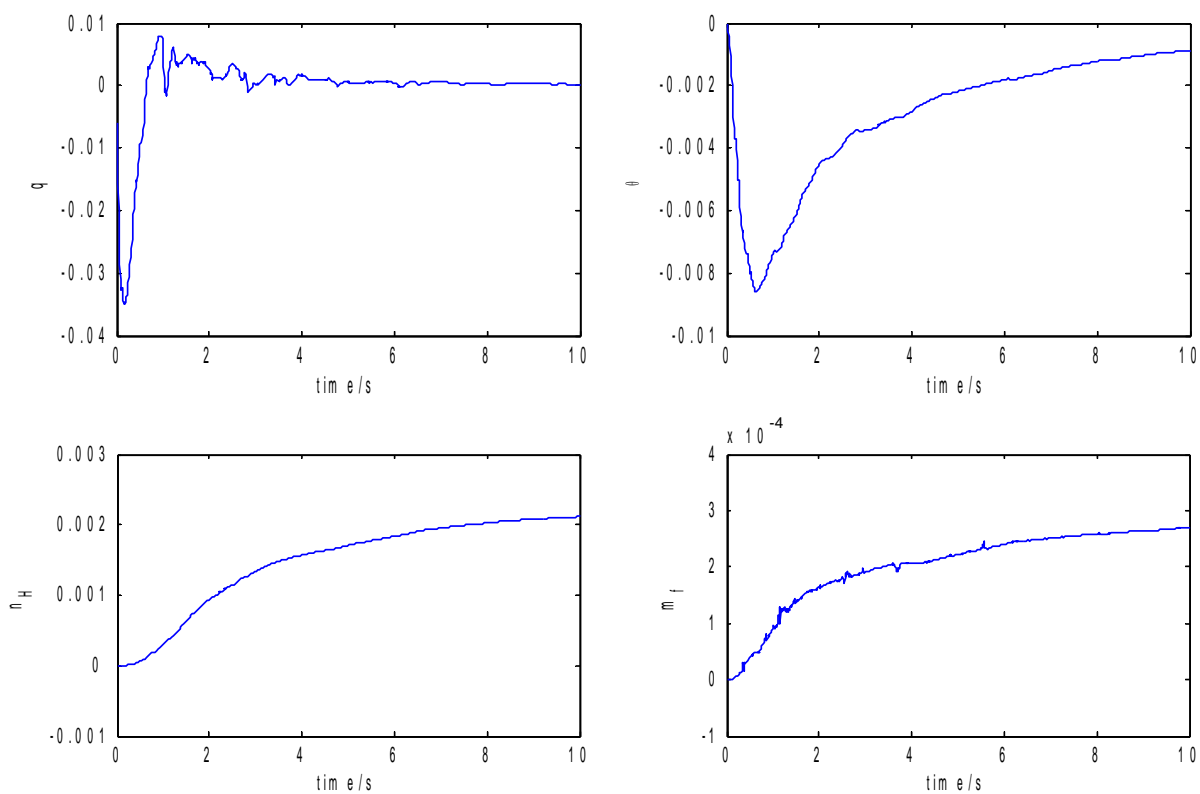

Figure 5 System step input response when the random time delay is $\tau \in[T, 2 T]$ and the packet dropout rate is 0.2 


\section{Conclusion}

First, the structure of a IFPC system was designed based on a certain type of aircraft and its propulsion system. Then, a Matlab/Simulink Truetime toolbox based DIFPC simulation platform is designed and the designing process is also given in the article. Finally, the effect of time delay and packet dropout on the system performance is carried out through the designed platform. The designed platform is advanced in its open access and easiness of extension and operation, which will provide an ideal simulation platform to the research of DIFPC system.

\section{References}

[1] Yangxu Zhao, Rui Ma. Review of integrate flight and propulsion Control Technology. Journal of Propulsion Technology. 20(1):108-111(1999)(In Chinese)

[2] Sanjay Garg, Duane L. Mattern, R. E. Bullard, Integrated Flight/Propulsion Control System Design Based on a Centralized Approach [J]. Journal of Guidance, Control and Dynamics. 14(1):107116(1991)

[3] James W. STOVL Integrated Flight and Propulsion Control - Current Successes and Remaining Challenges[C]. AIAA 2002-6021(2002)

[4] Mehrdad P, Marion M. Distributed Control of Turbofan Engines[C].AIAA-2009-5532(2009)

[5] Culley D. Plan for Revolutionary Change in Gas Turbine Engine Control System Architecture[R]. NASA -E-17833(2011)

[6] Peng Jing-bo, Zhang Zi-yang, Feng Feng. $H_{\infty}$ Optimal Control of Aero Engine Distributed Control System with Packet Dropout Compensator[J]. Procedia Engineering. 15(1):618-623(2011)

[7] Rock S M, Emami Naeini Abbas, Neighbors Ken. Integrated flight/propulsion Control:subsystem specifications[J]. Journal of Guidance, Control, and Dynamics. 17(1):201-208(1994)

[8] Yingqing Guo, Tongjun Tian. The Integrated Flight/Propulsion Controller Design for a New Fighter[J]. Journal of Aerospace Power. 18(4) :524-529(2003)(In Chinese) 